\section{ANÁLISE DO DESENVOLVIMENTO DE ELEMENTOS DO PENSAMENTO CIENTÍFICO EM ATIVIDADES DA DISCIPLINA FÍSICA APLICADA}

ANALYSIS OF SCIENTIFIC THINKING DEVELOPMENT IN ACTIVITIES FROM THE DISCIPLINE OF APPLIED PHYSICS

Douglas Carlos Vilela', Silvana Ligia Vincenzi²

\section{RESUMO}

Este trabalho teve como finalidade identificar e caracterizar elementos do pensamento científico em atividades de física da disciplina Física Aplicada com alunos do 3o semestre do curso Tecnólogo em Petróleo e Gás da Faculdade São Sebastião (FASS). Para isso, foi realizada uma microanálise etnográfica, que permitiu uma aproximação maior nas interações dos alunos durante as atividades. Foram gravadas em vídeo e áudio as aulas de laboratório onde os alunos realizaram essas atividades. Aplicou-se um questionário para verificar as práticas laboratoriais e as principais dificuldades encontradas pelos estudantes. Como conclusão, identificou-se que as atividades de modelagem de física possibilitam três momentos, nos quais foi perceptível o desenvolvimento de elementos do pensamento científico. Nesses momentos, identificaram-se todos os elementos: evidência, inferência, argumentação, análise e investigação. Os resultados encontrados no questionário mostraram que os alunos não tiveram prática 
Vilela DC, Vincenzi SL

Análise do desenvolvimento de elementos do pensamento científico em

atividades da disciplina Física Aplicada

laboratorial e nenhum contato com metodologia científica. Ademais, $46 \%$ dos universitários encontraram maior dificuldade na execução do relatório final.

Palavras-chave: Ensino de Física. Atividades experimentais. Pensamento científico.

\section{INTRODUÇÃO}

Atualmente, presenciam-se dificuldades no desenvolvimento de práticas laboratoriais, tanto no ensino médio como no ensino superior. Por outro lado, verifica-se um esforço de pesquisadores para buscar meios para se melhorar essa situação. E a experimentação pode ser uma metodologia motivadora do ponto de vista investigativo e que pode auxiliar no ensino-aprendizagem. Os experimentos podem servir de elo incentivador para que os estudantes dediquem-se às tarefas que são consideradas menos prazerosas e áridas, como a confecção de relatórios e conclusões de práticas, tornando o ensino agradável e com um retorno positivo na aprendizagem do aluno.

Dentro do panorama de atividades de laboratório, existe o desenvolvimento de práticas investigativas que permitem aos alunos agir e interagir, tanto com o fenômeno estudado como com os outros alunos.

A disciplina de Física Aplicada surge como uma ponte entre esse desenvolvimento intelectivo e as práticas de laboratório. 
Desse modo, este estudo traz as seguintes questões:

- Quais os elementos do pensamento científico que são possíveis de se identificar e se caracterizar na vivência dos alunos dentro da disciplina de Física Aplicada?

- Quais os tipos de atividades investigativas que permitem ações e interações dos alunos que manifestem o desenvolvimento de elementos do pensamento científico?

Nesse contexto, o presente trabalho propôs a análise do desenvolvimento de elementos do pensamento científico dos alunos durante as atividades investigativas de laboratório realizadas na disciplina de Física Aplicada do curso Tecnólogo em Petróleo e Gás da Faculdade São Sebastião (FASS), no ano de 2015.

A turma de Petróleo e Gás foi acompanhada durante um semestre com gravações em áudio e vídeo. Ademais, foram tiradas fotos e impressos relatórios como formas de avaliação do conteúdo abordado.

\section{REVISÃO BIBLIOGRÁFICA}

Este trabalho alicerça-se no pensamento científico. Borges (2006) ressalta como ocorre o ato do pensar científico:

Aprender o pensar científico é aprender a pensar uma situação nova ou não familiar, como o fazem mesmo aquelas pessoas com sólida formação 
Vilela DC, Vincenzi SL

Análise do desenvolvimento de elementos do pensamento científico em

atividades da disciplina Física Aplicada

numa certa área ou domínio do conhecimento. Elas avaliam o que sabem sobre aquilo e o que mais precisam saber, baseadas em seus conhecimentos, formulam hipóteses mesmo que precárias, avaliam que outras informações precisam obter para melhor compreender o problema, tratam de obter essas informações, tomando o cuidado de avaliar a sua origem, validade e confiabilidade (BORGES, 2006, p. 136).

Borges (2006) ainda destaca que o pensar científico desenvolvese quando se vivencia uma experiência investigativa mais autêntica.

Destaca-se a extensa obra de Kuhn $(1989,1991,1993,2001$, 2010 e 2016), que, num esforço de décadas, estudou as interações de sala de aula para identificar e caracterizar as habilidades do pensamento científico existentes em situações escolares.

Avalia-se que o laboratório escolar pode propiciar excelentes oportunidades para desenvolver o pensamento científico dos estudantes (MILLAR, 1996; HODSON, 1993; AAAS, 1990).

As atividades promovidas nos laboratórios devem ser diversificadas e balanceadas, estimulando o desenvolvimento dos alunos. Dentre tantas atividades, acredita-se que investigações de variados níveis de complexidade, propostas pelos alunos ou pelo professor, têm o potencial de engajar e motivar os estudantes. Além disso, elas também permitem a superação das deficiências das atividades práticas tradicionais e fazem com que os estudantes tenham um papel mais ativo no processo de aprendizagem (AAAS, 1990). 
No entanto, Millar e Osbourne (1998) destacam alguns elementos que julgam importantes na formação do aluno e que devem, sim, ser estimulados por parte do docente. Por exemplo: saber a prática científica e ter elementos para se posicionar diante do contexto nos quais os alunos vivem. Ainda observa-se a importância de o professor desenvolver a argumentação e a explicação como parte dos atributos que os alunos devem aprimorar para usarem futuramente em suas vidas (MILLAR; OSBOURNE, 1998).

Dessa forma, tanto Millar e Osbourne (1998) como Santos e Mortimer (2000) exploram o momento de aprendizagem em ciências com as formas de pensar, isto é, argumentar, investigar, formar um modelo mental de um fenômeno.

Ancorado nisso, há a visão de que, dentro de uma sala de aula, existem alunos com diferentes opções e aptidões para as mais variadas áreas, como: agricultura, biotecnologia, comunicação, medicina, dentre outras, que necessitam, além de profissionais, cientistas, para ampliarem os estudos das demais áreas.

Em seu trabalho, Kuhn (2010) descreve o que é e como se desenvolve o pensamento científico. Para tal, ela define o pensamento científico como uma atividade humana intrínseca na maioria das pessoas, assim como a inferência e a resolução de problemas. Em particular, a autora destaca um alinhamento entre a argumentação e a experimentação como característica do pensamento científico (KUHN, 1993; 2010). 
Vilela DC, Vincenzi SL

Análise do desenvolvimento de elementos do pensamento científico em

atividades da disciplina Física Aplicada

Kuhn (2010) ainda define que o pensamento científico é de natureza social, ocorre muito por meio da interação social em um grupo de pessoas.

Uma das caracterizações parte da relação do pensamento científico, como a coordenação entre a teoria e as evidências. Para a autora, existe uma nítida diferença entre teoria e evidência. Com relação à teoria, os alunos reivindicam do professor conhecer as definições de um assunto específico. Ao passo que, nas evidências, os alunos buscam num fenômeno ou num problema as informações para compreender a situação.

A segunda caracterização de Kuhn (2010) destaca que haverá um aprimoramento do conhecimento científico dos alunos no momento em que eles se questionarem quanto ao próprio pensamento.

As origens do pensamento científico podem ser definidas em três processos, de acordo com Kuhn (2010): diferenciar afirmação de inferência, identificar a evidência como fonte de conhecimento e desenvolver a coordenação entre teoria e evidência como um processo contínuo.

Destaca-se a recente produção de Kuhn (2016), na qual a autora traz uma análise de aprendizagem baseada em problemas como uma proposta positiva para o desenvolvimento de habilidades do pensamento científico.

As ações identificadas, no geral, são: investigação, análise, inferência, argumentação e evidência. Consideram-se essas ações como pertencentes ao pensamento científico. Tem-se finalmente construído o alicerce teórico. 
E há outros trabalhos que também tratam do pensamento científico como formado por duas dimensões: a dos conhecimentos de domínio específico, que englobam conceitos, modelos e teorias próprios de cada uma das ciências, e a das estratégias de domínio geral, que abordam a elaboração e o teste de hipóteses, a coordenação entre teoria e evidência, a elaboração de inferências, a construção de modelos etc. (ZIMMERMAN, 2000, 2007; BORGES, 2006; KUHN, 2010).

Interessante destacar que as pesquisas com relação ao desenvolvimento do pensamento científico são apresentadas em momentos distintos. No ensino fundamental (ZIMMERMAN, 2007; KUHN, 2010) e também para o ensino médio (ZIMMERMAN, 2000; BORGES, 2006). Ainda em tempo, houve um grande direcionamento para esse estudo no ensino superior, principalmente no que se refere à formação de professores (BORGES, 2006), atingindo, assim, o ciclo de formação científica desde o fundamental até o ensino superior.

A partir dessa grande linha condutora que é o pensamento científico, o Quadro 1 apresenta as fases do pensamento científico adotadas neste trabalho (KUHN, 2010). A partir desses elementos é que se podem analisar as ações e interações dos alunos. 
Vilela DC, Vincenzi SL

Análise do desenvolvimento de elementos do pensamento científico em

atividades da disciplina Física Aplicada

Quadro 1 - Elementos do pensamento científico definido nesta pesquisa com base nas definições de Kuhn $(1998,2001$, 2006, 2010)

\begin{tabular}{|c|}
\hline Elementos do pensamento científico \\
\hline Investigar \\
\hline Analisar \\
\hline Inferir \\
\hline Argumentar \\
\hline Evidenciar \\
\hline
\end{tabular}

Fonte: adaptado de Kuhn (2010).

\section{METODOLOGIA}

Como delineamento metodológico, trata-se de uma pesquisa de natureza aplicada, sendo classificada, quanto à abordagem do problema, como qualitativa e quantitativa (MARCONI; LAKATOS, 2010).

A abordagem qualitativa foi interpretativista da pesquisa, de cunho etnográfico, em que os dados foram coletados por meio de áudio e vídeo, a partir de gravadores e câmeras digitais. $\mathrm{Na}$ parte quantitativa, foram coletados dados por meio de um questionário, contendo quinze itens e analisados pela estatística descritiva. Este trabalho foi desenvolvido na turma de disciplina de Física Aplicada do curso de Petróleo e Gás da Faculdade São Sebastião (FASS), que é composta por quinze alunos.

Assim, para distinguir as interações aluno-atividade, alunoprofessor, focou-se no conjunto de aulas gravadas da disciplina 
de Física Aplicada e nas notas de campo, para que fosse possível organizar e identificar as situações de ações e interações em grupo. Gravações de áudio, notas de campo e elementos do pensamento científico foram utilizados para identificar os eventos relevantes, ou seja, as situações em que os alunos apresentam potencial para desenvolver os elementos do pensamento científico. Nas interações aluno-atividade, os alunos presenciaram, durante as práticas experimentais, situações que possibilitaram que se relacionassem com as seguintes ações: investigar, modificar, identificar, descrever e manipular.

Buscou-se identificar, nas ações e interações, manifestações de elementos como: análise de dados, inferências sobre fenômenos e resultados, arguição sobre ações realizadas no desenvolvimento do experimento físico e discussão dos experimentos e dificuldades derivadas deles.

Para organizar essa gama de informações, preparou-se um mapa de eventos para cada aula, com os dados relacionados às interações coletivas, tendo como referência as gravações em áudio. Nesses mapas de eventos, registraram-se, no formato de tabela: uma coluna com o tempo de gravação; uma coluna com as atividades coletivas e as interações entre professor e aluno - ou entre aluno e aluno -; outra coluna com as atividades específicas dos grupos de alunos; e finalmente uma coluna com observações e caracterizações das ações ou interações que mereciam destaque, segundo a avaliação do pesquisador.

O mapeamento dos eventos teve grande importância para o momento de análise das interações dos alunos. Por meio 
Vilela DC, Vincenzi SL

Análise do desenvolvimento de elementos do pensamento científico em

atividades da disciplina Física Aplicada

desses documentos, foi possível reunir elementos para se tornar esclarecedora a forma como foram produzidos os relatórios.

Procurou-se explorar de maneira abrangente os dados coletados, para se conseguir avançar com relação à descrição de eventos, produzindo-se novos entendimentos sobre o desenvolvimento de elementos do pensamento científico, por meio de ações e interações em aulas de Física Aplicada.

\section{RESULTADOS E DISCUSSÃO}

A seguir, têm-se as aulas consideradas mais pertinentes para as análises de manifestações de interações que representam o desenvolvimento do pensamento científico por parte dos alunos.

As aulas selecionadas foram duas de quatro horas cada. $\mathrm{Na}$ primeira delas, foi realizada a modelagem do sistema hídrico da FASS pelos alunos. A segunda foi uma aula de laboratório em que os estudantes analisaram a hidrodinâmica (escoamento) de diferentes fluidos em tubulações de superfícies distintas.

Respeitando as proposições da pesquisa, optou-se pela utilização de codinomes para os alunos, visando preservar as identidades deles (Igor, João, Mário, Gustavo, Maria, Leonardo, Luiz, Nelson, Rosa, Rodolfo, Babi, César, Sílvio, Sérgio e Ana). 


\section{Modelagem do sistema hídrico}

Nessa aula, foi solicitado aos alunos que realizassem uma análise do sistema hídrico da Faculdade São Sebastião (FASS). A proposta era tirar fotos de cada um dos andares da faculdade que possuísse sanitários e bebedouros.

Posteriormente, de posse dessas informações, os alunos realizariam uma planta $2 \mathrm{D}$ e uma planta $3 \mathrm{D}$, representando as tubulações de água e esgoto.

\section{Momento 1 - Identificando uma grandeza física}

como parte da solução

Igor: “Maria, como vamos passar uma tubulação por baixo da outra se ambas estão na altura do chão? Vai fazer um furo no isopor?"

Maria: “Não sei. Se a gente fizer um sobre o outro?"

Igor: “Mas ele não é sobreposto."

Sílvio: "E se fizer uma legenda de qual é água e qual é esgoto?"

Maria: "Isso precisa mesmo, usamos cores distintas pra tubulação de água e outra para esgoto."

Igor: "E tem que mostrar a altura, lembra lá da questão de energia, a água vai com pressão por causa da altura da caixa d'água. Precisamos mostrar isso aqui."

Rosa: "E se usar aquele canudinho com curva só pra demonstrar que está sobreposto?" 
Vilela DC, Vincenzi SL

Análise do desenvolvimento de elementos do pensamento científico em

atividades da disciplina Física Aplicada

Igor: "Pode ser, vamos mostrar que não estão no mesmo lugar e precisamos medir isso aí, precisa estar em proporção com as fotos."

\section{Momento 2 - Análise de proporções}

Mário: "Qual a altura que eu coloco a tubulação de entrada e saída do esgoto? Tem uma diferença aqui. Vocês mediram?"”

Babi: "Deu menos de $10 \mathrm{~cm}$.

Mário: "Quanto é menos de $10 \mathrm{~cm}$, Babi?"

Babi: "Vou ver aqui, tá no caderno."

Mário: "E quanto é isso na nossa escala? Alguém faz essa conta, e eu já passo pra maquete."

João: "1 metro é 10 cm."

Babi: “Deu 8 cm, Mário."

Mário: “Tá, então, isso dá 8 mm de canudo, certo?"

João, Babi: “Certo."

Mário: "Tem que manter a proporção, senão a planta (referência à planta 2D) fica diferente da maquete."

\section{Momento 3 - Investigação sobre o escoamento da tubulação de esgoto}

Sérgio: "Se a gente deixar os canos do esgoto em linha reta significa que ele está assim no banheiro, mas tem uma queda, pra escoar pra caixa de esgoto." 
Vilela DC, Vincenzi SL

Análise do desenvolvimento de elementos do pensamento científico em atividades da disciplina Física Aplicada

César: "Sim, todo banheiro tem uma queda pro ralo também."

Ana: "Mas esse não precisa pôr, precisa?"

Sérgio: “Não, só a da caixa do esgoto."

Gustavo: "Tem uma caixa lá fora, dá pra medir a distância."

César: "O importante é a altura. Lembra da energia."

Ana: "É verdade, energia potencial."

Gustavo: "Mas ele desce em linha reta? Às vezes, desce torto?"

Ana: "Como assim torto? (risos)."

Gustavo: "Uma parte reta assim e outra, assim" (mostrando com a mão, vertical e horizontal).

Ana: "Ah, pode ser mesmo. Mas, ainda assim, o importante é altura. Vai que tem um tubo desnivelado, né?"

Sérgio: "Vamos medir isso lá?"

César: "A gente pode jogar água e ver quanto tempo demora pra chegar à caixa."

Gustavo: "Mas o que você quer achar com isso?"

César: "Dá pra calcular, cronometra e mede o tamanho."

Ana: "Mas a gente quer saber o desnível."

Sérgio: "Vamos lá fazer mais medidas."

Gustavo, Ana: "Vamos." 


\section{Análise qualitativa}

No primeiro momento, identifica-se a manifestação de alguns elementos do pensamento científico: evidência, inferência e argumentação.

A evidência mostrou-se presente quando os estudantes perceberam a relação da altura e que esta precisa ser representada na maquete, uma vez que são perceptiveis os desníveis de altura entre as tubulações nas fotos. A partir de um material (foto), os alunos levantaram as evidências e buscaram as soluções.

No processo de busca da solução, a inferência, a análise e a argumentação se demonstraram presentes. Os estudantes colocaram em pauta alguns fatores que poderiam ser úteis, como cores de canudos, furos na maquete e curvas dos canudos. Essa manifestação é caracterizada como inferência, isto é, quando são colocadas para discussão algumas soluções possíveis.

A discussão em si é uma forma de argumentação, na qual cada um apresentou os porquês de se utilizar a inferência proposta. Como na explicação sobre a questão energética, em que há relação com a altura, feita por Igor (MOMENTO 1).

No segundo momento, manifestaram os seguintes elementos do pensamento científico: evidência e análise.

A evidência fez-se presente quando Mário traz a dúvida sobre a altura do esgoto com relação à proporção que foi definida pelo grupo anteriormente. Seguindo essa evidência, surge 
a análise, quando os estudantes confirmam os valores, e Mário confirma a proporção de 10 centímetros de canudo para 1 metro da medida real. Nesse momento, percebe-se a manifestação do elemento de análise, em que Mário verifica com os outros estudantes um valor.

Não foi possível identificar outros elementos no segundo momento.

No terceiro momento, identifica-se a manifestação de todos os elementos do pensamento (evidência, inferência, análise, argumentação e investigação).

Identifica-se a evidência, elemento do pensamento científico, nos momentos em que os alunos percebem alguns atributos que causam neles o levantamento de hipóteses, como, por exemplo, o desnível do ralo e a caixa de esgoto fora da faculdade.

A análise é sugerida pelos estudantes a partir do momento em que eles percebem esse desnível para escoamento do esgoto. $E$, por conseguinte dessa análise, surgem as inferências, como a forma de escoamento, se essa acontece em linha reta, se é torta ou se tem queda de água. Todas essas inferências são debatidas com argumentos.

Os argumentos surgem a partir de algumas grandezas, como a altura da tubulação. Os estudantes também utilizaram o desnível da tubulação como argumentação para interpretar o problema de escoamento. Finalmente, eles partem para a investigação, quando Sérgio propõe medir a altura da caixa do esgoto. 
Vilela DC, Vincenzi SL

Análise do desenvolvimento de elementos do pensamento científico em

atividades da disciplina Física Aplicada

Em tempo, não se pode dizer que a sequência dos elementos do pensamento científico identificados nos momentos analisados apresenta linearidade ou sequência, como aqui foi apresentado.

Eles surgiram dessa forma íntegra, e não se procura encontrar um padrão na ocorrência dos fatos. Isto é, não significa que foi o levantamento de evidências que levou à proposta de investigação. Afinal, a investigação ocorreu no Momento 3, no entanto não esteve presente nos dois primeiros momentos.

Dessa forma, o Quadro 2 mostra os elementos encontrados em cada momento escolhido da atividade de modelagem. Percebe-se que, no terceiro momento, foi possível desenvolverse um número maior de elementos, dada a complexidade da situação. Ao passo que o segundo momento não permitiu melhor elaboração, restringindo-se apenas a dois elementos. Com relação ao laboratório experimental, os dados obtidos não foram exatos, muito menos coesos, para se calcular a energia. Dessa forma, a análise será colocada.

Quadro 2 - Elementos do pensamento científico identificados durante as interações na atividade de modelagem hídrica

\begin{tabular}{|c|c|}
\hline Momento & Elementos \\
\hline Momento 1 & $\begin{array}{c}\text { Evidência, inferência e } \\
\text { argumentação }\end{array}$ \\
\hline Momento 2 & Evidência e análise \\
\hline Momento 3 & $\begin{array}{c}\text { Evidência, inferência, análise, } \\
\text { argumentação e investigação }\end{array}$ \\
\hline
\end{tabular}

Fonte: elaborado pelos autores, 2016. 


\section{Análise quantitativa}

A partir do questionário aplicado, foi possível identificar os momentos em que houve maior dificuldade por parte dos alunos e, a partir disso, confrontá-los com os dados obtidos em áudio e vídeo.

O questionário foi dividido em duas partes. A primeira delas foi de caráter pessoal, em que foram perguntadas algumas características, como: idade, gênero e procedência escolar.

O resultado obtido foi que o público-alvo, isto é, os alunos do terceiro semestre de Petróleo e Gás da FASS, possuem idade entre 19 e 30 anos. É majoritariamente masculino (80\%). E todos os alunos vieram de escola pública (100\%).

Na segunda parte, foram realizadas perguntas com relação à prática experimental e como eles a desenvolveram (escoamento de fluidos).

A primeira questão versava sobre a relação das aulas teóricas em sala de aula com as aulas de laboratório. Em sala de aula, foram realizadas três aulas expositivas sobre energia mecânica e cinética. Posteriormente, foram realizadas duas aulas de laboratório, visando a esse conteúdo, porém associado aos fluidos, isto é, energia mecânica dos fluidos.

O Gráfico 1 apresenta a opinião dos alunos com relação às aulas teóricas e práticas. 
Vilela DC, Vincenzi SL

Análise do desenvolvimento de elementos do pensamento científico em

atividades da disciplina Física Aplicada

Gráfico 1 - Opinião dos alunos com relação às aulas teóricas e práticas de energia mecânica

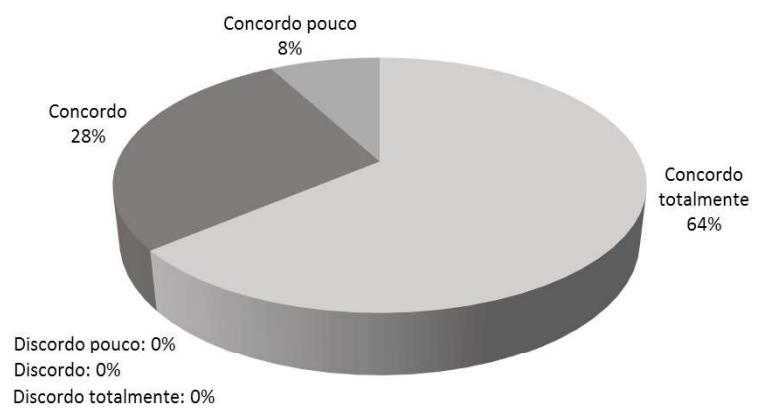

Fonte: elaborado pelos autores, 2016.

Percebe-se que a maioria dos alunos (64\%) concorda totalmente com a relação das aulas teóricas com as práticas. Ao passo que $28 \%$ concordam e $8 \%$ concordam pouco e ninguém discordou dessa questão.

A prática experimental deve ser realizada concomitantemente com as aulas teóricas, para que seja possível realizá-las de uma maneira mais coordenada, como preconizam Zompero e Laburú (2011). Pozo e Crespo (2009) também salientam que as atividades investigativas devem ser precedidas por alguma exposição teórica ou, ao menos, científica, pois, ao proporcionar uma situação nova, os alunos devem ter alicerce para saber realizá-las.

O Gráfico 2 mostra a percepção dos alunos com relação às grandezas físicas dos fluidos. Durante a exposição em sala de aula, foi salientado que existem grandezas que são distintas nos fluidos - viscosidade, densidade, massa -, dessa forma, 
esperava-se que os alunos percebessem essas grandezas durante a análise dos fenômenos.

Gráfico 2 - Possível percepção dos alunos a respeito da relação das grandezas físicas no experimento de escoamento de fluido

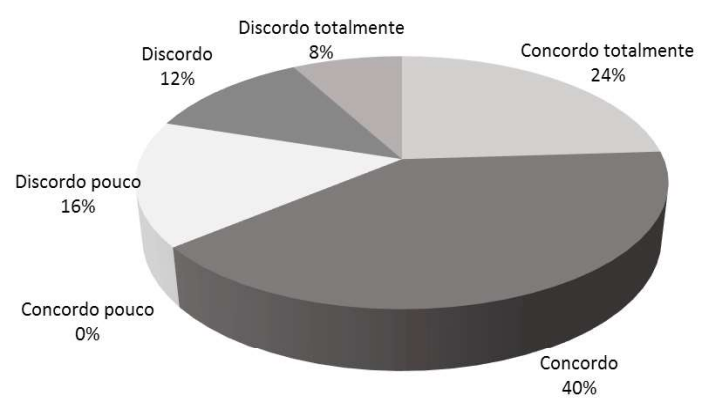

Fonte: elaborado pelos autores, 2016.

Percebe-se que a maior parte dos alunos (40\%) concorda e $24 \%$ concordam totalmente que o experimento possibilitou perceber a distinção entre as grandezas do fluido. Todavia, também há um número expressivo que discordou pouco (16\%), que discordou (12\%) ou discordou totalmente (8\%).

McDermott e Shaffer $(2001,2010)$ alerta, em seus trabalhos, que há uma distância entre o que se ensina e o que se aprende em situações laboratoriais. A mesma autora ainda complementa que mostrar a situação para os alunos não significa que eles vão interpretá-las como os professores as interpretam, mas, sim, que é primordial proporcionar a eles situações para que investiguem o fenômeno, e não apenas para que observem algo pronto. 
Vilela DC, Vincenzi SL

Análise do desenvolvimento de elementos do pensamento científico em

atividades da disciplina Física Aplicada

No Gráfico 3, temos a questão em que os alunos apresentam opiniões com relação à dificuldade na execução do relatório. Percebe-se, no Gráfico 3, que 92\% encontraram muita dificuldade na execução, pois concordaram plenamente. De fato, isso foi perceptível. Mesmo com o longo prazo para a execução do relatório, a pouca vivência com essa situação mostrou-se evidente, uma vez que os alunos cometiam equívocos em excesso.

Gráfico 3 - Dificuldade encontrada pelos alunos na execução do relatório

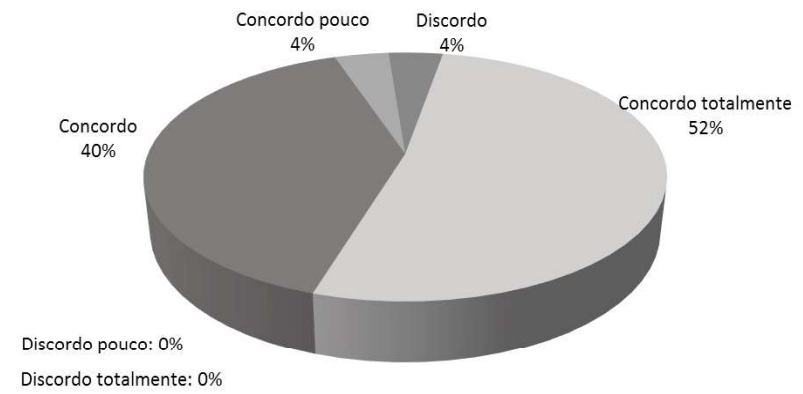

Fonte: elaborado pelos autores, 2016.

O Gráfico 4 apresenta os momentos em que os alunos encontraram maior dificuldade na realização das atividades. 
Gráfico 4 - Opiniões acerca de quais foram as dificuldades encontradas

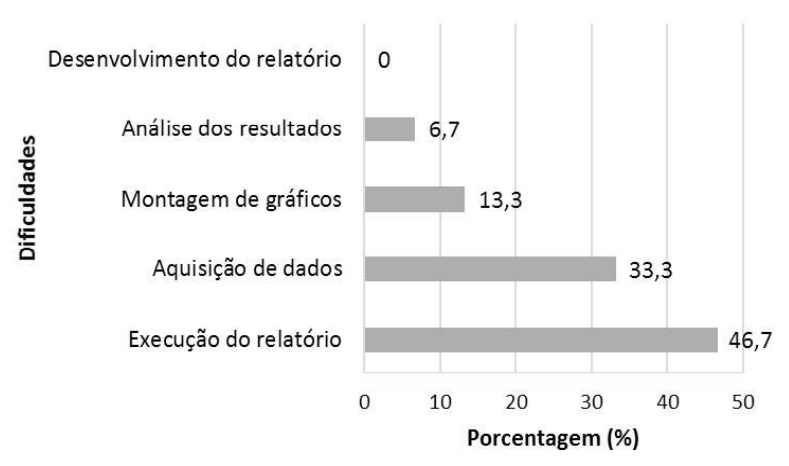

Fonte: elaborado pelos autores, 2016.

Por fim, observa-se, no Gráfico 4, que as atividades em que os alunos encontraram mais dificuldades na prática foram: a execução do relatório $(46,7 \%)$ juntamente com a aquisição de dados (33,3\%), ambas são práticas metodológicas. Esses resultados ratificaram que os alunos mostraram pouca ou nenhuma vivência laboratorial e metodológica e, assim, tiveram dificuldades para organizar e interpretar os dados. Consequentemente, essas situações geraram dificuldades no desenvolvimento do relatório, uma vez que são etapas que antecederam o desenvolvimento dele.

Dessa forma, finalizam-se as questões realizadas, percebendose já alguns motivos para tantas dificuldades encontradas nas realizações das práticas e dos relatórios. Essa análise será apresentada com mais profundidade nas considerações finais. 


\section{CONCLUSÕES}

As considerações finais iniciam-se resgatando o objetivo desta pesquisa, que foi identificar elementos do pensamento científico em atividades de física e que tipo de atividades permitem esse desenvolvimento.

Conclui-se que os objetivos deste trabalho foram atendidos e o que se segue nestas considerações são todos os dados obtidos a partir da pesquisa e como eles corroboram para o ensino de Física.

A partir das aulas analisadas, conseguiu-se identificar três principais momentos durante as práticas investigativas nas quais foi possível caracterizar, nas interações dos alunos, desenvolvimento de elementos do pensamento científico. Foram eles: o Momento 1 - Identificando uma grandeza física como parte da solução, o Momento 2 - Análise de proporções e o Momento 3 - Investigação sobre o escoamento da tubulação de esgoto.

Em cada um desses momentos, foi possível identificar elementos que, segundo Kuhn (2010), são informações que compõem o pensamento científico e que se julga serem importantes para o desenvolvimento em atividades de ciências, segundo Borges (2006).

Percebe-se que, no terceiro momento, foi possível encontrar mais elementos do pensamento científico. Entende-se que a atividade do Momento 3 tem características mais complexas, o que levou à maior decorrência de ações proporcionando esse desenvolvimento. 
O elemento da investigação mostrou-se importante na discussão de grandezas físicas, essa sendo o alvo final da aprendizagem laboratorial de Física.

Outro elemento interessante foi a argumentação, quando os alunos discutiam entre si as alturas e os desníveis. Ao discutirem o conteúdo, eles estão revendo e pensando sobre alternativas e possibilidades comparadas a um pensamento inicial.

Por outro lado, as aulas práticas mostraram, do modo como foram realizadas, que em nada podem contribuir para o desenvolvimento do pensamento científico.

Os alunos apresentaram poucas ações e interações. Quando houve, as ações eram dúvidas com relação a procedimentos e cálculos a serem efetuados, não se caracterizando como pensamento científico. Uma possibilidade para esse fato ter ocorrido é a inexperiência dos estudantes com relação a práticas laboratoriais. É possível que, se os alunos tivessem habilidades básicas, surgissem as interações almejadas nesta pesquisa.

Com relação ao questionário, percebeu-se que os alunos têm defasagem completa no ensino científico. Todos os estudantes não tiveram aula de metodologia científica, na qual é possível aprender as normas básicas para elaboração de relatórios, bem como as etapas de uma pesquisa científica.

Em decorrência dessa defasagem, observou-se uma grande dificuldade na coleta de dados, na participação e no envolvimento na atividade laboratorial. 
Vilela DC, Vincenzi SL

Análise do desenvolvimento de elementos do pensamento científico em

atividades da disciplina Física Aplicada

Destaca-se, ainda, o fato de os relatórios apresentarem muita discrepância quanto à análise dos dados. Durante as aulas de modelagem hídrica, percebeu-se um conteúdo de discussão muito mais elaborado se comparado com os dos relatórios apresentados. A maior dificuldade encontrada pelos alunos foi o desenvolvimento do relatório.

O desfecho deste trabalho, a partir dessas informações, mostra que as atividades de modelagem têm potencial de desenvolvimento dos elementos do pensamento científico. As atividades de laboratório sem prática prévia em nada contribuem para o desenvolvimento do pensamento científico. Os elementos que estiveram mais presentes nas ações e interações dessa análise foram evidência, inferência e argumentação.

Este trabalho endossa as pesquisas que vêm sendo feitas com relação ao pensamento científico e às práticas laboratoriais por Borges (2006) e Zompero e Laburú (2011), no âmbito nacional.

No âmbito internacional, esta pesquisa contribui com o campo de ensino de ciências sob o aspecto de análise de atividades de ensino superior e reflexões acerca do desenvolvimento de elementos do pensamento científico já estudados por autores como Kuhn (2010).

\section{REFERÊNCIAS}

AAAS. Science for All Americans: Project 2001. New York: Oxford University Press, 1990. 
BORGES, Oto. Formação inicial de professores de Física: Formar mais! Formar melhor! Revista Brasileira de Ensino de Física, v. 28, n. 2, p. 135-142, 2006.

HODSON, D. Re-thinking old ways: towards a more critical approach to practical work in school science. Studies in Science Education, v. 22, p. 85-142, 1993.

KUHN, D. Children and adults as intuitive scientists. Psychological Review, 96, p. 674-689, 1989.

KUHN, D. The skills of argument. New York: Cambridge University Press, 1991.

KUHN, D. Science as argument: implications for teaching and learning scientific thinking. Science Education, 77, p. 319-337,1993a.

KUHN, D. Connecting scientific and informal reasoning. Merrill-Palmer Quarterly, 39, p. 74-103, 1993b.

KUHN, D. How do people know?. Psychological Science, 12, p. 1-8, 2001.

KUHN, D. What is scientific thinking and how does it develop? In: GOSWAMI, Usha. Blackwell handbook of childhood cognitive development. Blackwell Publishers: Malden, MA, USA, 2010.

KUHN, D. Social science as a tool in developing scientific thinking skills in underserved, low-achieving urban students. Journal of Experimental Child Psychology, v. 143, mar. 2016, p. 154-161.

MARCONI, M. A.; LAKATOS, E. M. Fundamentos de metodologia científica. 7. ed. São Paulo: Atlas, 2010. 
Vilela DC, Vincenzi SL

Análise do desenvolvimento de elementos do pensamento científico em

atividades da disciplina Física Aplicada

MCDERMOTT, Lillian; SHAFFER, Peter. Preparing teachers to teach Physic and Physical Science by inquiry. Physics Education, SI, 2010.

MCDERMOTT, Lillian; SHAFFER, Peter. Tutorials in introductory Physics. New York: Prentice Hall, 2001.

MILLAR, R. Towards a science curriculum for public understanding. School Science Review, v. 77, n. 280, p. 7-18, 1996.

MILLAR, R.; OSBORNE, J. Beyond 2000: Science education for the future. London: King's College, 1998.

POZO, J. I.; CRESPO, M. A. G. A aprendizagem e o ensino de ciências: do conhecimento cotidiano ao conhecimento científico. 5. ed. Porto Alegre: Artmed, 2009.

SANTOS, W. L. P.; MORTIMER, E. F. Uma análise de pressupostos teóricos da abordagem C-T-S (CiênciaTecnologia-Sociedade) no contexto da educação brasileira. Ensaio Pesquisa em Educação em Ciências, v. 2, n. 2, p.133-162, 2000.

ZIMMERMAN, C. The development of scientific reasoning skills. Developmental Review, v. 20, n. 1, p. 99-149, mar. 2000.

ZIMMERMAN, C. The development of scientific thinking skills in elementary and middle school. Developmental Review, v. 27, n. 2, p. 172-223, jun. 2007.

ZIMMERMAN, C. Development of science process skills in the early childhood years. In: CABE TRUNDLE, K.; SAÇKES, M. (Eds.). Research in early childhood science education. Dordrecht: Springer, 2015. p. 143-165. 
ZOMPERO, A. F.; LABURÚ, C. E. Atividades investigativas no ensino de ciências. Rev. Ensaio, Belo Horizonte, v. 13, n. 3, p. 67-80, 2011.

\section{ABSTRACT}

This paper had the purpose to identify and characterize elements of scientific thinking in applied physics activities from an Oil \& Gas industry undergraduate course with students in the third period. To conduct such investigation, ethnographic microanalysis was used, allowing close approach of students' interaction. Audio and video recordings were made in laboratories where activities were conducted. We also applied a survey questionnaire to assess laboratory practices and the major difficulties students might face. In conclusion, modeling activities allowed three moments, in which the development of scientific thinking elements was perceptible. In those moments, the following elements were identified: evidence, inference, argument, analysis and investigation. Findings demonstrate that students had had no laboratory practice or contact with scientific methodology before starting university. In addition to that, final paper writing was chosen by $46 \%$ of the university students as the most difficult activity.

Keywords: Physics teaching. Laboratory activities. Scientific thinking. 
Vilela DC, Vincenzi SL

Análise do desenvolvimento de elementos do pensamento científico em

atividades da disciplina Física Aplicada

\section{Douglas Carlos Vilela}

Doutorando em Física pelo Instituto Tecnológico de Aeronáutica (ITA). Ex-professor de Física Aplicada e Matemática Financeira na Faculdade São Sebastião (FASS/UNICSUL). Físico licenciado pela UFSCar. Especialista em Ensino de Ciências pela UTFPR. Mestre em Educação em Ciências e Matemática pela UFSCar.

douglascarlosvilela@gmail.com

\section{Silvana Ligia Vincenzi}

Professora titular e pesquisadora da Universidade Tecnológica Federal do Paraná (UTFPR), campus Medianeira. Atualmente é professora do Programa de Pós-Graduação em Tecnologias Computacionais para o Agronegócio, atuando na linha de pesquisa Tecnologias Computacionais Aplicadas à Produção Agrícola (UTFPR/MD), e professora do Programa de PósGraduação em Métodos e Gestão em Avaliação (UFSC).

sligie@globo.com 